\title{
PROVA TUBERCULINICA, BCG ORAL E INFECÇÃO TUBERCULOSA EM CRIANÇAS MENORES DE 5 ANOS.
}

\author{
Marialda Höfling de Pádua Dias* \\ Anita Hayashi *
}

RSPUB9/432

\begin{abstract}
Dias, M. H. de P. \& Hayashi, A. Prova tuberculinica, BCG oral e infecção tuberculosa em crianças menores de 5 anos. Rev. Saúde públ., S. Paulo, 12: 443-54, 1978.

Resumo: São relatados os resultados das provas tuberculinicas com PPD Rt23, 2 UT, em crianças menores de um ano e de um a 4 anos, matriculadas na Clinica Pediátrica do Hospital das Clínicas da Faculdade de Medicina da USP, São Paulo, Brasil, no periodo de 1971 a 1975. Em 665 crianças menores de um ano encontrou-se 3,15\% de reatores fracos e 6,62\% de reatores fortes e em 1.298 crianças de um a 4 anos, $0,69 \%$ de reatores fracos e 5,5\% de reatores fortes. Nas mesmas crianças, foram estudadas as relações entre vacinação BCG oral prévia e positividade à prova tuberculinica nos 2 grupos etários considerados $e$ nos quais se obteve a informação de vacinação anterior com BCG oral. Em 575 crianças menores de um ano e 1.113 de um a 4 anos encontrou-se associação positiva entre vacinação $B C G$ oral prévia e positividade à prova tuberculínica. Analisando a relação entre o número de doses de BCG oral prévio e o resultado das provas tuberculinicas pelo método de Goodman, verificou-se que a proporção de crianças que tinham tomado 3 doses e mais de BCG oral e que apresentaram reação forte da prova tuberculinica é significantemente maior que a observada para os não reatores, fato esse não verificado para o grupo de um a 4 anos. Nas crianças que tomaram uma ou duas doses não foram encontradas diferenças estatisticamente significantes.
\end{abstract}

UnITERmos: Tuberculose infantil. Teste tuberculínico. Vacinação BCG oral.

\section{T TRODUCG A}

A prova tuberculínica padronizada pela Organização Mundial de Saúde (OMS) tem sido empregada no Brasil, a partir de 1961, para detectar a presença de infecção tuberculosa, principalmente no grupo etário de menores de 15 anos de idade. A sua importância em Saúde Pública tem sido inestimável, pois permite através da medida de prevalência da infecção tuberculosa, conhe- cer a tendência e a magnitude do problema da tuberculose numa população ${ }^{20}$.

A maioria das pesquisas sobre prevalência da infecção tuberculosa no nosso pais foi realizada em escolares $1,2,9,16,17,18,19,20,21,25$ em virtude da facilidade de operacionalização para se atingir essa faixa etária. Seria também importante o conhecimento da prevalência de infecção em crianças de mais

* Do Instituto da Criança do Hospital das Clínicas da Faculdade de Medicina da USP Av. Dr. Enéas de Carvalho Aguiar, 255 - 05403 - São Paulo, SP - Brasil. 
DIAS, M. H. de P. \& HAYASHI, A. Prova tuberculínica, BCG oral e infecçáo tuberculosa em crianças menores de 5 anos. Rev. Saude puibl., S. Paulo, 12:443-54, 1978.

baixa idade não só pela sua maior suscetibilidade em relação à tuberculose, mas também porque ela reflete a existência de individuos baciliferos no micro ambiente dessa população ${ }^{\circ}$.

A dificuldade de operacionalização para se atingir esse grupo e a escassez de recursos disponiveis tornam esse procedimento inexequivel em larga escala. Assim, são raros os dados representativos da real situação em nosso meio, no que se refere à infecção tuberculosa antes da idade escolar. Na maioria das vezes eles são obtidos não de estudos populacionais mas de instituições onde se utiliza a prova tuberculínica como rotina na detecção da infecção tuberculosa. Contudo, na quantificação da infecção tuberculosa, não se tem levado em consideração a vacinação BCG oral prévia. Sabe-se que uma proporção de individuos considerados infectados pelo bacilo da tuberculose é representada por vacinados previamente com BCG oral, fato esse verificado por alguns autores quer experimentalmente em animais ${ }^{3,24}$ quer em pesquisas no ser humano $1, \pm, 19$.

Os estudos realizados relacionando vacinação BCG oral e alergia tuberculínica pósvacinal não foram, contudo, conclusivos, pairando ainda muitas dúvidas sobre a real capacidade dessa vacina provocar e manter a alergia através dos anos.

Em vista desses fatos, achamos oportuno estudar a infecção tuberculosa em crianças menores de um ano e de um a 4 anos e analisar a relação entre vacinação BCG oral prévia e positividade à prova tuberculínica.

\section{MATERIAL E METODOS}

A população de estudo é constituída por crianças menores de um ano e de um a 4 anos que, no período de $1^{\circ}$ de janeiro de 1971 a 31 de dezembro de 1975, demandaram a Clínica Pediátrica do Hospital das Clínicas da Faculdade de Medicina da USP e que, tendo sido matriculadas nessa clinica, foram submetidas à prova tuberculinica. A população de menores de um ano é composta de 665 crianças e a de um a 4 anos, de 1.298 crianças. Essa população corresponde, respectivamente, a $22,31 \%$ e $43,54 \%$ da população total de zero a 14 anos, admitidas no serviço, no período de tempo considerado.

As provas tuberculínicas foram realizadas segundo técnica padronizada pela OMS \&. A tuberculina empregada foi o PPD Rt 23 2UT $(0,04 \mathrm{mcg} / 0,1 \mathrm{ml})$ fabricada pelo "Statens Seruminstitut" de Copenhagen e diluído pela Unidade de Tuberculina do Laboratório de Referência da Campanha Nacional contra a Tuberculose (CNCT). As provas foram realizadas por uma auxiliar de enfermagem treinada e padronizada, utilizando-se seringas, agulhas e réguas milimetradas conforme requisitos exigidos pela OMS.

A prova foi lida em milimetros após $72 \mathrm{~h}$ e, segundo o maior diâmetro transverso da induração, o resultado foi interpretado em não reator $=0$ a $4 \mathrm{~mm}$; reator fraco $=$ $5-9 \mathrm{~mm}$ e reator forte $=10 \mathrm{~mm}$ e mais 8 . Foram consideradas reatoras à prova tuberculínica as crianças que apresentaram $5 \mathrm{~mm}$ e mais de induração.

Os dados levantados foram: idade, número de prontuário, data de aplicação da prova e da leitura, número de doses e clata de recebimento do $B C G$ oral, informação esta obtida diretamente da mãe ou responsável pela criança.

$\mathrm{Na}$ segunda etapa do trabalho, só foram relacionadas as crianças que tinham informação de vacinação anterior com $B C G$ oral e que tinham realizado a prova tuberculínica pelo menos 2 meses após a administração da vacina. Por esse motivo a população ficou ređuzida a 575 crianças menores de um ano e 1.113 de um a 4 anos (Tabelas 2 e 3 ).

Para a análise estatística foram utilizados testes de associação pelo método do $\mathrm{X}^{2}$ com correção para continuidade para determinar as associaçōes significantes ao nivel de $5 \%$ e cálculo do coeficiente de Yule para medir o grau de associação existente 
DIAS, M. H. de P. \& HAYASHI, A. Prova tuberculinica, BCG oral e infecção tuberculosa em crianças menores de 5 anos. Rev. Saúde públ., S. Paulo, 12:443-54, 1978.

entre as variáveis em estudo e, contrastes de Goodman ${ }^{12}$ que permitem discriminar diferenças de proporção.

\section{RESULTADOS E DISCUSSÃO}

\section{1 - Resultados das Provas Tuberculinicas}

A Tabela 1 mostra as proporções de crianças não reatoras, reatoras fracas e reatoras fortes à prova tuberculínica nas três faixas etárias.

Por essa Tabela, podemos observar que $9,8 \%$ das crianças menores de um ano são reatoras à prova tuberculínica, proporção essa que está muito próxima daquela encontrada por Certain e col. ${ }^{10}$ em crianças da área de Pinheiros, referente ao Dispensário da Faculdade de Saúde Pública da USP, no periodo de 1960 a 1969 e é menor daquela encontrada pelo mesmo autor na área do bairro do Ipiranga referente ao Dispensário da Associação dos Sanatórios Populares "Campos de Jordão". Entretanto, no trabalho de Certain e col.10, de 1960 a 1967, foi utilizado o PPD Rt23 1UT e de 1968 em diante, 2 UT. Já no estudo de Nunes ${ }^{15}$, no Município de Osasco, contíguo a cidade de São Paulo, essa proporção é de $12,1 \%$ e se deve exclusivamente aos reatores fracos à prova tuberculínica. No grupo etário de um a 4 anos a proporção encontrada de $6,24 \%$ de reatores é muito próxima daquelas de Certain e col. ${ }^{11}$ nos dois estudos referidos e tem uma diferença de $2,1 \%$ a mais daquela encontrada no Municipio de Osasco ${ }^{15}$.

Comparando a proporção de reatores fracos à prova tuberculínica por nós encontrada com aquela obtida nas pesquisas de Certain e col. ${ }^{10}$ e Nunes ${ }^{15}$, verificamos que a proporção de reatores fracos é sempre maior no grupo de menores de um ano. Esse fato nos leva a pensar na possibilidade de maior influência da vacição oral prévia sobre a sensibilidade tuberculínica no primeiro ano de vacinação. Passos Filho ${ }^{19}$, estudando a viragem tuberculínica pós-vacinal, obteve uma média de $20,90 \%$ de reatures tracos após 51 a 90 dias ou 91 a 120 dias de vacinação em recém-nascidos, enquanto que Almeida ${ }^{1}$ obteve, em escolares, cerca de $16,3 \%$ de reatores fracos após 90 dias de vacinação.

Um aspecto que chama a atenção é o fato de termos encontrado proporção maior de reatores fortes, tanto em menores de um ano como enı crianças de um a 4 anos, comparada com os achados dos autores citados. Por outro lado, a proporção de reatores fortes $(6,6 \%)$ nos menores de um ano, muito próxima daquela descrita por Certain e col. ${ }^{9}(\mathbf{7}, 1 \%)$ em escolares da primeira série da Rede Municipal de Ensino do Município de São Paulo, no periodo de 1971 a 1974, sugere a existência de infecção tuberculosa numa fase precoce da vida e uma maior probabilidade de existência de foco tuberculoso no micro-ambiente das crianças que são matriculadas no nosso serviço. Entretanto, uma proporçào de reatores fortes à prova tuberculínica pode também refletir vacinação $B C G$ oral prévia como já foi relatado por Passos Filho e col. ${ }^{19}$ e Almeida e col. ${ }^{1}$, em trabalhos experimentais com $B C G$ e sensibilidade tuberculínica pós-vacinal em crianças $100 \%$ vacinadas. No nosso trabalho, entretanto, entre as crianças com informação de BCG anterior, apenas $51,13 \%$ no grupo de menores de $\mathrm{um}$ ano e $50,0 \%$ no de $\operatorname{lm}$ a 4 anos tinhan recebido $\mathrm{BCG}$ oral.

\section{Prova Tuberculinica e BCG oral prévio}

Das 665 crianças menores de um ano e das 1.298 crianças de um a 4 anos, 13,52\% e $14,25 \%$, respectivamente, não tinham informaçōes sobre BCG oral prévia. Considerando, portanto, somente as 1.688 crianças com informação sobre vacinação anterior com BCG, a relação entre vacinação e positividade à prova tuberculínica foi analisada sob dois aspectos: um, analisando a vacinação $B C G$ oral prévia e a positividade à prova tuberculínica, e outro analisando a relação entre o número de doses de BCG e a reação à prova tuberculínica. 
DIAS, M. H. de P. \& HAYASHI, A. Prova tuberculínica, BCG oral e infeção tuberculosa em crianças menores de 5 anos. Rer. Saúde públ., S. Paulo, 12:443-54, 1978.

TA B E L A 1

Resultados da prova tuberculínica (PPD Rt23 2UT) em crianças menores de 5 anos, segundo grupos de idade e tamanho da induração. Ambulatório de Pediatria - Hospital das Clinicas da Faculdade de Medicina da USP - S. Paulo, 1971 - 1975.

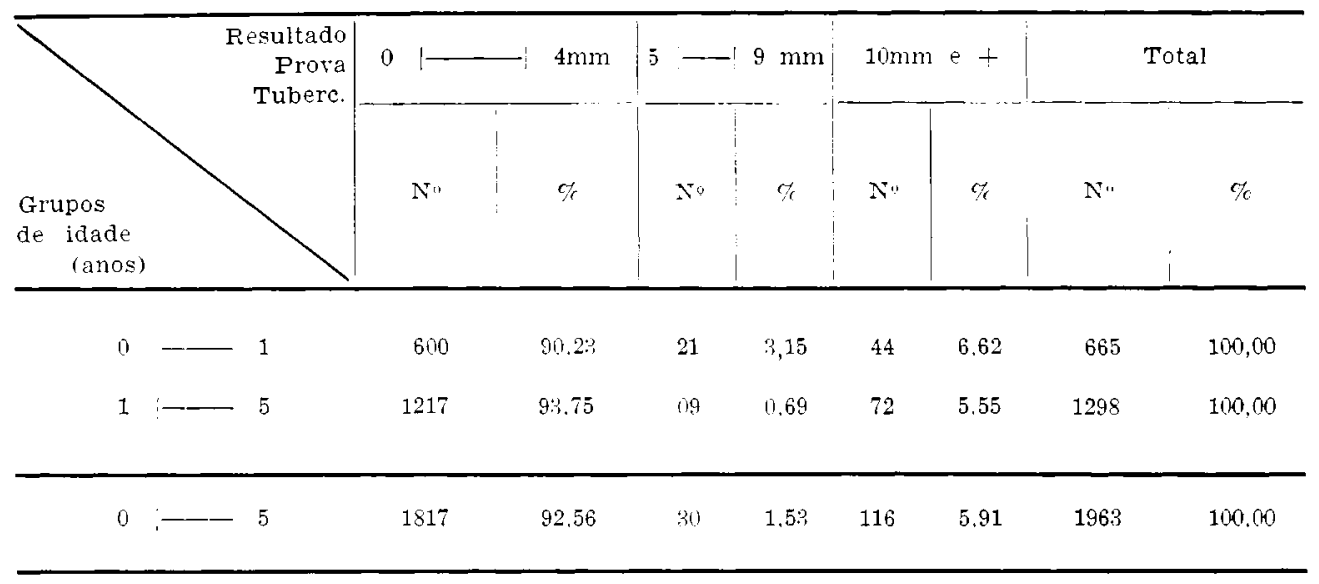

T A B E L A 2

Distribuição de reatores e não-reatores à prova tuberculinica (PPD-Rt23-2UT) segundo vacinação BCG oral préria em menores de um ano. Ambulatório de Pediatria - Hospital das Clinicas - Faculdade de Medicina da USP - S. Paulo, 1971 - 1975

\begin{tabular}{|c|c|c|c|}
\hline Resultado & $\begin{array}{l}\operatorname{Sim} \\
(\%)\end{array}$ & $\begin{array}{l}\text { Não } \\
(\%)\end{array}$ & $\begin{array}{c}\text { Total } \\
(\%)\end{array}$ \\
\hline Reator & $\begin{array}{c}57 \\
(19,39)\end{array}$ & $\begin{array}{c}3 \\
(1,07)\end{array}$ & $\begin{array}{c}60 \\
(10,43)\end{array}$ \\
\hline Não-reator & $\begin{array}{c}237 \\
(80,61)\end{array}$ & $\begin{array}{c}278 \\
(98,93)\end{array}$ & $\begin{array}{c}515 \\
(89,57))\end{array}$ \\
\hline Total & $\begin{array}{c}294 \\
(100,00)\end{array}$ & $\begin{array}{c}281 \\
(100,00)\end{array}$ & $\begin{array}{c}575 \\
(100,00)\end{array}$ \\
\hline
\end{tabular}

$\mathrm{X}_{2}$ obs. $=49,66$

coef. Yule $=0,91$

\subsection{Análise da relação entre vacinação} $B C G$ oral prévia e prova tuberculinica

As Tabelas 2, 3 e 4 mostram os resultados da prova tuberculinica segundo vacinação $B C G$ oral prévia, respectivamente, em crianças menores de um ano, de um a 4 anos e de zero a 4 anos.
A análise estatística dessas tabelas pelo teste do $\mathrm{X}^{2}$ com correção para a continuidade, tendo como $\mathrm{X}^{2}$ crítico $\lg 1 \alpha=0,05$ $=3,84$, revelou que existe associação positiva entre vacinação BCG oral prévia e reação positiva à prova tuberculínica. 
DIAS, M. H. de P. \& HAYASHI, A. Prova tuberculínica, BCG oral e infeç̧ão tuberculosa em crianças menores de 5 anos. Rev. Saúde públ., S. Paulo, 12:443-54, 1978.

TA B E L A 3

Distribuição de reatores e não-reatores à prova tuberculínica (PPD-Rt23-2UT), segundo vacinação $B C G$ oral prévia em crianças de 1 - 5 anos. Ambulatório de Pediatria Hospital das Clínicas - Faculdade de Medicina da USP - S. Paulo, 1971 - 1975.

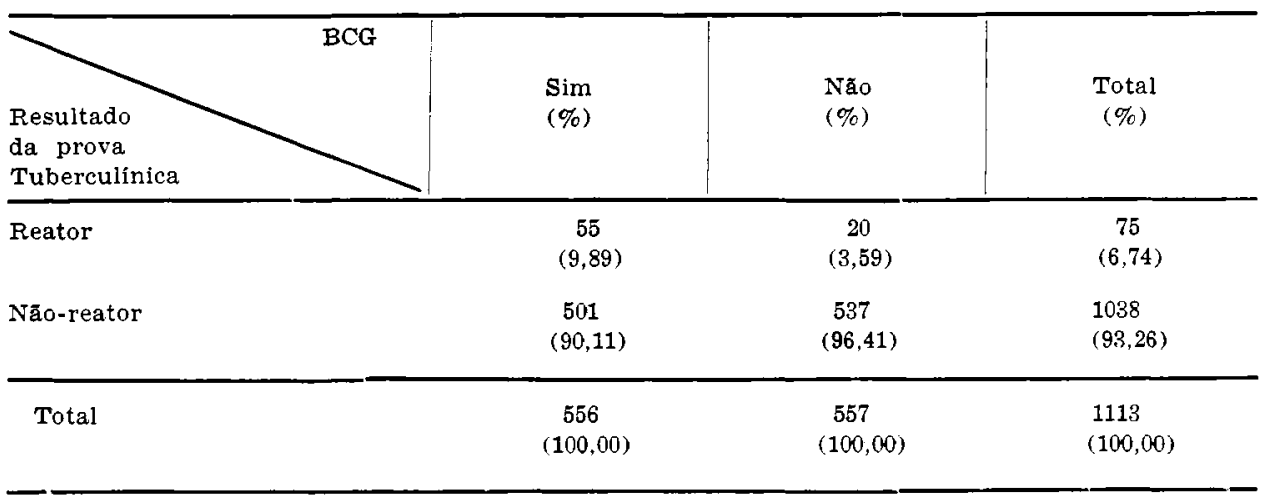

$\mathrm{X} 2$ obs. $=16,59$

$\mathrm{X} 2$ crit. 1 gl $\alpha=0,05=3,84$

coef. Yule $=0,60$

T A BE L A 4

Distribuição de reatores e não-reatores à prova tuberculínica (PPD Rt23 2UT), segundo vacinação BCG oral prévia em ctianças de 0 - 5 anos. Ambulatório de Pediatria Hospital das Clínicas - Faculdade de Medicina da USP - S. Paulo, 1971 - 1975.

\begin{tabular}{|c|c|c|c|}
\hline $\begin{array}{l}\text { Resultado } \\
\text { da prova } \\
\text { Tuberculinica }\end{array}$ & $\begin{array}{l}\operatorname{Sim} \\
(\%)\end{array}$ & $\begin{array}{l}\text { Não } \\
(\%)\end{array}$ & $\begin{array}{l}\text { Total } \\
(\%)\end{array}$ \\
\hline Reator & $\begin{array}{c}112 \\
(13,18)\end{array}$ & $\begin{array}{c}23 \\
(2,74)\end{array}$ & $\begin{array}{c}135 \\
(8,00)\end{array}$ \\
\hline Não-reator & $\begin{array}{c}738 \\
(86,82)\end{array}$ & $\begin{array}{c}815 \\
(97,26)\end{array}$ & $\begin{array}{c}1553 \\
(92,00)\end{array}$ \\
\hline Total & $\begin{array}{c}850 \\
(100,00)\end{array}$ & $\begin{array}{c}838 \\
(100,00)\end{array}$ & $\begin{array}{c}1688 \\
(100,00)\end{array}$ \\
\hline
\end{tabular}

$\mathrm{X} 2$ obs. $=61,00$

coef. Yule $=0,69$

$\mathrm{X} 2$ crit, 1 gl $\alpha=0,05=3,84$

Esse mesmo fato foi também observado por Loureiro e col.17, em crianças de zero a 14 anos, em uma área de Salvador.

Podemos verificar também, pela Tabela 2 , que dentre as crianças menores de um ano que tomaram BCG oral previamente, $19,39 \%$ apresentaram reação positiva à prova tuberculínica. No grupo etário de um a 4 anos, e no de menores de 5 anos, nas mesmas condições acima mencionadas, as proporçōes foram de $9,89 \%$ e $13,18 \%$, respectivamente (Tabelas 3 e 4 ).

Esses achados diferem daqueles de Passos Filho e col.19 que encontraram uma média de $35,19 \%$ de reações positivas após 51 a 90 dias ou 91 a 120 dias da aplicação 
DIAS, M. H. de P. \& HAYASHI, A. Prova tuberculinica, BCG oral e infecção tuberculosa em crianças menores de 5 anos. Rev. Saúde públ., S. Paulo, 12:443-54, 1978.

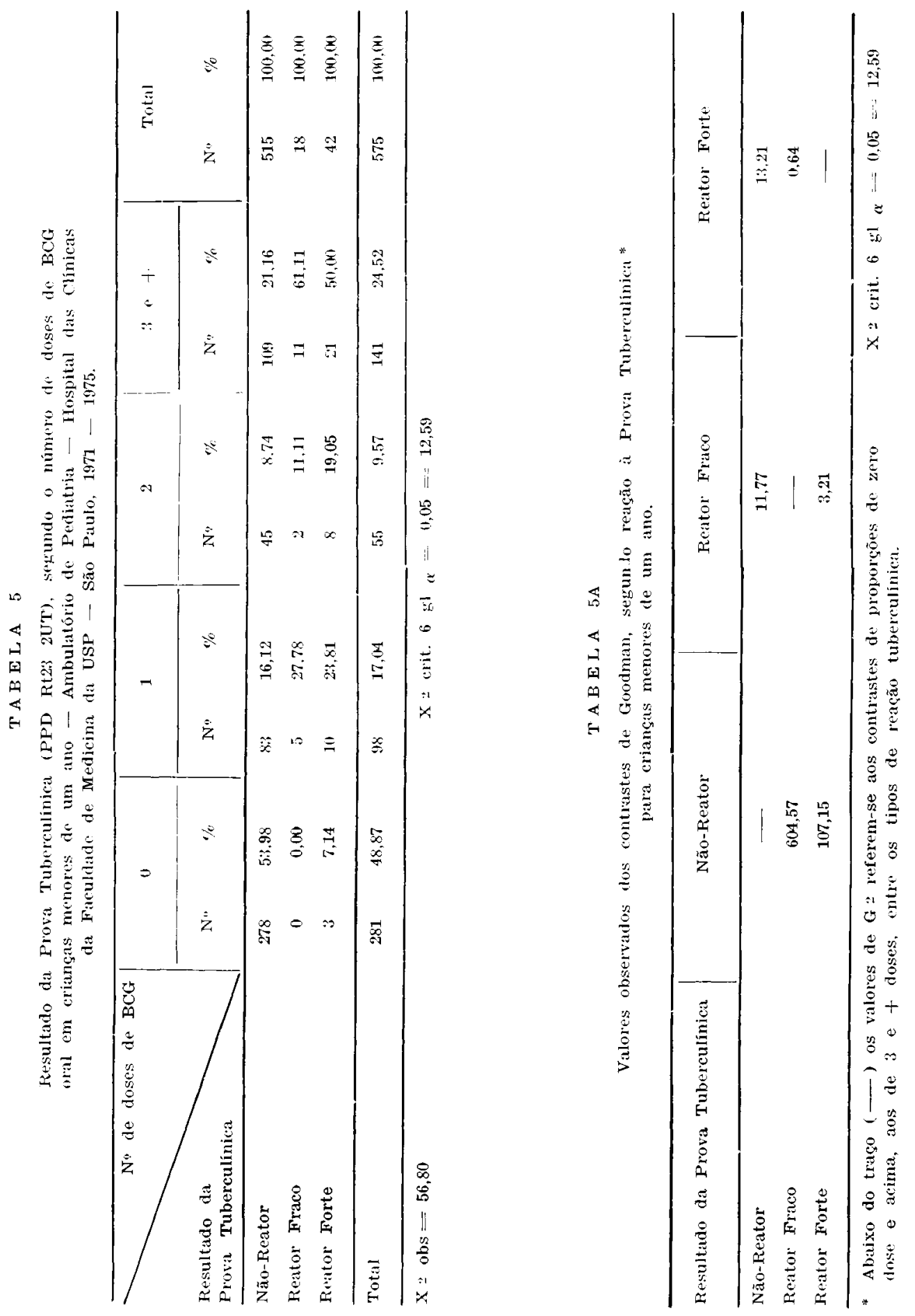


DIAS. M. H. de P. \& HAYASHI, A. Prova tuberculínica, BCG oral e infecção tuberculosa em crianças menores de 5 anos. Rev. Saúde públ., S. Paulo, 12:443-54, 1978.

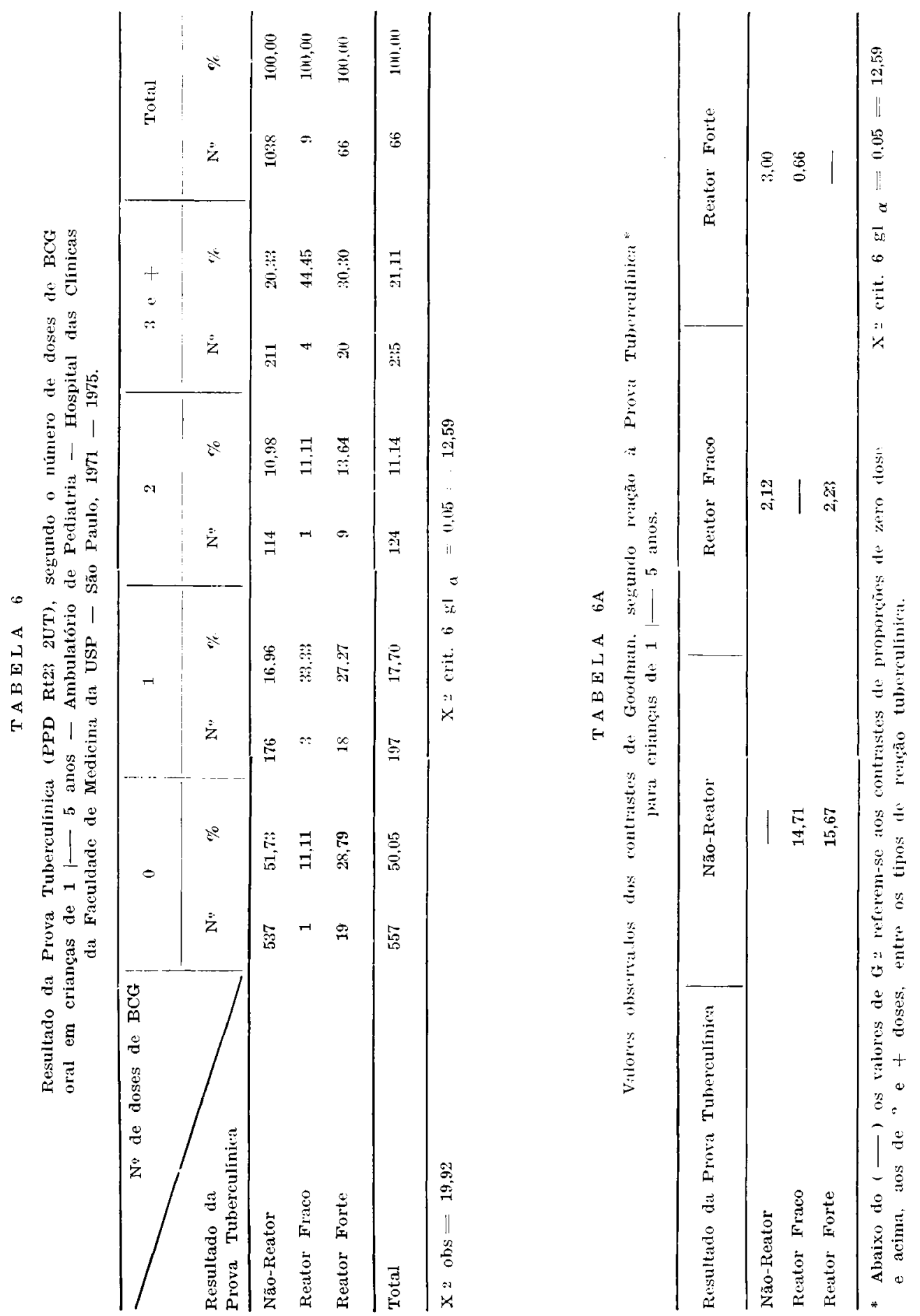


DIAS, M. H. de P. \& HAYASHI, A. Prova tuberculínica, BCG oral e infecção tuberculosa em crianças menores de 5 anos. Rev. Saúde públ., S. Paulo, 12:443-54, 1978.

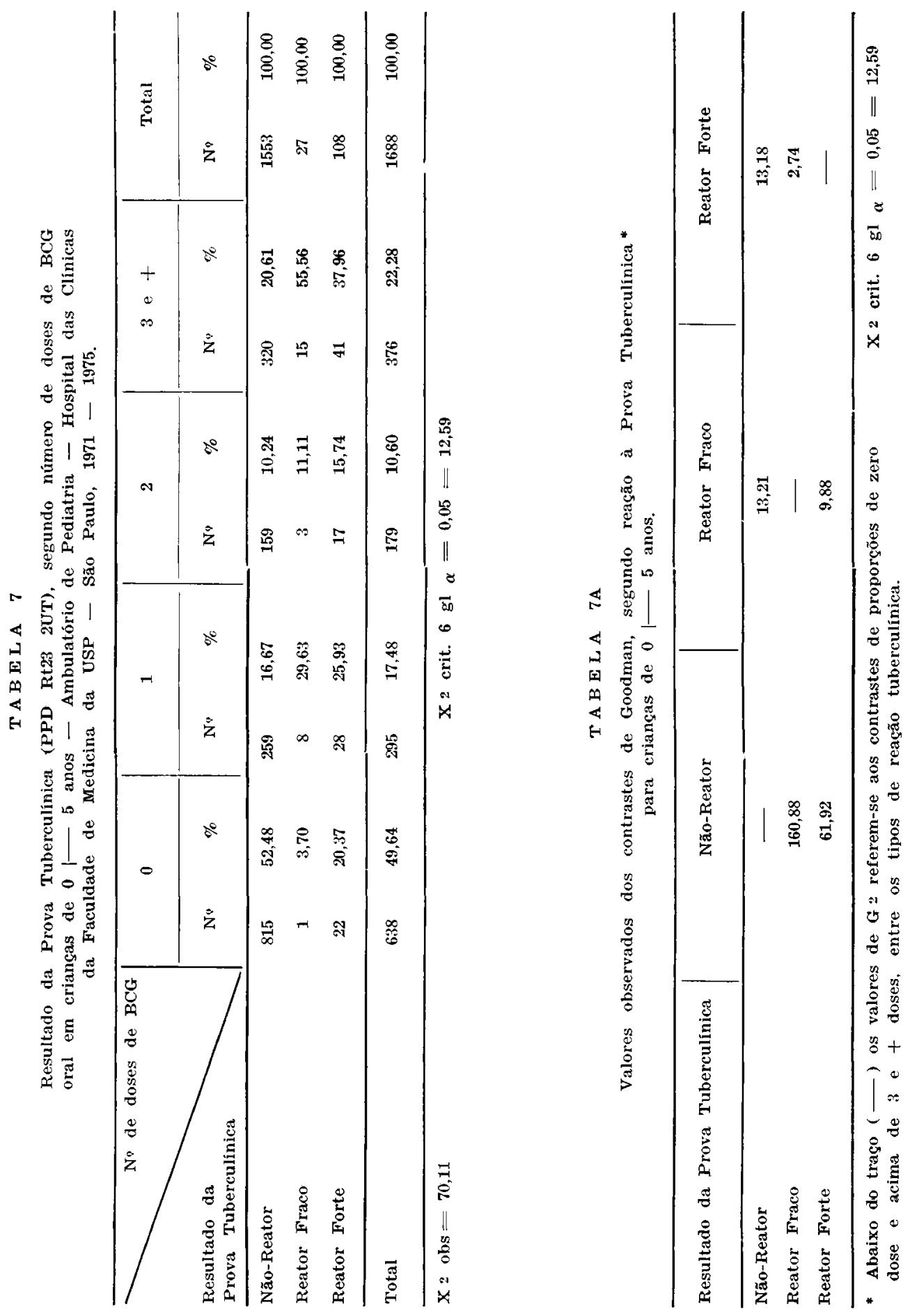


DIAS, M. H. de P. \& HAYASHI, A. Prova tuberculínica, BCG oral e infecção tuberculosa em crianças menores de 5 anos. Rev. Saúde públ., S. Paulo, 12:443-54, 1978.

da vacina em recém-nascidos. Diferem também daqueles de Brólio e col. 7 que encontraram $29,4 \%$ de reatores à prova tuberculínica em crianças previamente vacinadas com BCG oral.

Resultados semelhantes aos nossus, no grupo de menores de um ano, foram relatados por Kahn e col.14 que encontraram $19 \%$ de positividade à prova tuberculínica aos 2 meses de idade, en crianças oralmente vacinadas com BCG por ocasião do nascimento.

$\mathrm{Na}$ tentativa de explicar essas diferenças, pensamos nas seguintes eventualidades:

- A positividade da reação diminuiria conı o tempo, fato já relatado por vários pesquisadores, inclusive Assis 5, que em dados apresentados em 1940 já observava a diminuição gradativa da proporção de reatores a partir dos 4 meses até 48 meses de idade, em crianças vacinadas ao nascimento.

- A vacinação não teria obedecido às nurmas de sua aplicação.

\subsection{Análise da relação entre resultado da} prova tuberculinica e número de doses de BCG oral prévio.

Nas Tabelas 5,6 e $\mathbf{7}$ foram colocados resultados da prova tuberculínica, segundo o número de doses de $\mathrm{BCG}$ oral prévio.

Quando nos referimos ao grupo das crianças que tomaram 3 e mais doses de $B C G$ oral, na realidade, esse grupo se compõe em sua maioria de crianças que tomaram 3 doses, pois apenas 3 crianças menores de um ano, e 11 de um a 4 anos haviam tomado mais de 3 doses.
Aplicando 0 teste do $\mathrm{X}^{2}$, adotando 0 nível de significância de $5 \%$ e 6 graus de liberdade $\left(\mathrm{X}^{2}\right.$ crítico $\left.6 \mathrm{gl} \alpha=12,59\right)$, pudemos verificar que havia associação entre o número de doses de BCG e a reação tuberculínica nos três grupos (Tabelas 5, 6 e 7 ).

Empregando o teste de contrastes de Goodman ${ }^{12}$ verificamos o número de doses que seria capaz de discriminar a diferença de proporções. Os contrastes de proporção são representados por $\mathrm{G}^{2}$ que se comparam com $0 \mathrm{X}^{2}$ crítico.

Para os grupos etários de menores de um ano, de um a 4 anos, e de zero a 4 anos, analisados distintamente, obtivemos os seguintes resultados: a proporção dos que não tomaram BCG previamente dentre os que não apresentaram reação à prova tuberculínica é significantemente maior que aquelas observadas entre os que apresentaram reação fraca e forte, Tabelas $5 \mathrm{~A}$, $6 \mathrm{~A}$ e $7 \mathrm{~A}$, respectivamente.

A análise das diferenças de proporções de uma e duas doses de BCG não apresentou significância estatística entre as categorias de reação à prova tuberculínica, nos 3 grupos etários considerados.

Ao analisarmos a proporção de 3 e mais doses de BCG, verificamos que para os menores de um ano, a proporção é maior entre os que apresentaram reação forte do que aquela observada entre os não reatores.

Para as crianças de um a 4 anos, a proporção de 3 e + doses de BCG não apresentou diferença entre as categorias de tipo de reação à prova tuberculínica, porém, no grupo de zero a 4 anos, a proporção de 3 e mais doses de BCG é maior 
DIAS, M. H. de P. \& HAYASHI, A. Prova tuberculínica, BCG oral e infecção tuberculosa em crianças menores de 5 anos. Rev. Saúde públ., S. Paulo, 12:443-54, 1978.

entre os reatores fortes comparada com a de não-reatores. Logicamente isto se deve à influência do grupo de menores de um ano, visto não se ter encontrado significância no grupo de crianças de um a 4 anos.

Para explicar esse fato, poderianos pensar na possibilidade de atenuação da sensibilidade tuberculínica influenciada pelo tempo decorrido após vacinação, como Assis e Carvalho s já descreveram em 1940, utilizando Tuberculina Velha na concentração de 1:100 e 1:50, e Amaral e Sorensen ${ }^{3}$ encontraram experimentalmente em cobaios utilizando Tuberculina Velha a $1: 10$. Essa atenuação que se acentuaria com o decorrer do tempo, poderia ser a responsável pelos nossos achados, se considerarmos que a maioria das crianças, no nosso meio, recebiam BCG oral na época do nascimento. Entretanto, os achados da literatura são controvertidos, pois Sénécal ${ }^{23}$ refere manutenção da sensibilidade tuberculínica em $80,5 \%$ de casos, de 15 meses a 5 anos e meio da viragem tuberculínica ocorrida após administração de BCG oral, a crianças de zero a 4 anos, utilizando duas doses de $200 \mathrm{mg}$, uma dose de 400 e uma de 650 $\mathrm{mg}$, pelo "método brasileiro". Entretanto, as doses de $400 \mathrm{mg}$ e $650 \mathrm{mg}$ não são empregadas, de rotina, no Brasil.11. Para essas doses de BCG, Sénécal 23 não encontrou diferença de manutençăo de sensibilidade tuberculinica, estatisticamente significantes, não referindo, porém, a concentração e o tipo de tuberculina empregada.
Mesmo em relação ao BCG intra-dérmico, cuja capacidade de promover sensibilidade tuberculínica pós-vacinal tem sido amplamente estudada, permanecem muitas dúvidas a respeito da duração dessa sensibilidade tuberculinica, em virtude das discrepâncias existentes entre diferentes autores 13,22 .

\section{CONCLUSOEES}

1. O indice de infecção tuberculosa traduzido pela proporção de reatores fortes à prova tuberculinica é relativamente elevado para crianças menores de um ano e de um a 4 anos, comparado com outros achados no nosso meio.

2. Houve associação positiva entre vacinação $B C G$ oral prévia e reação positiva à prova tuberculinica.

3. A vacinação BCG oral prévia realizada com 3 doses e + da vacina pode ser responsável por uma proporção de reatores fortes à prova tuberculínica nas crianças menores de um ano.

\section{AGRADECIMENTOS}

A Maria Lúcia de Moura Silva Soboll, pela valiosa assessoria estatística e à D. Maria Paul, Auxiliar de Enfermagem do mesmo Instituto, pela realização das provas tuberculínicas. 
DIAS, M. H. de P. \& HAYASHI, A. Prova tuberculínica, BCG oral e infecção tuberculosa em crianças menores de 5 anos. Rev. Saúde públ., S. Paulo, 12:443-54, 1978.

DiAS, M. H. de P. \& HAYASHI, A. [Tuberculin test, oral BCG vaccine, and tuberculosis infection among children under five years of agel Rev. Sañde públ., S. Paulo, $12: 443-54,1978$.

ABSTRACT: Results of tuberculin reaction from PPD Rt 23, 2UT are reported on children under one year of age and children from one to four years of age who were registered in the Pediatric Clinics of the Hospital das Clinicas of the College of Medicine of the State University of Säo Paulo. The study was carried out from 1971 through 1975. In a group of 665 children under one year of age, $3.15 \%$ were weak reactors while $6.62 \%$ were strong reactors, and, in a group of 1298 children between one to four years of age, $0.69 \%$ were weak reactors while $5.5 \%$ were strong reactors. The relationship between prior $B C G$ oral vaccination and positivation to the tuberculin test in the two age groups was studied, thus obtaining information about the previous oral BCG vaccination. Likewise, in 575 children under one year of age and 1113 children one to four years of age, a positive relationship between the previous ora! administration of $B C G$ and the positivation to the tuberculin test was found. In analyzing the relationship between the number of doses of previous oral BCG administration and the results of the tuberculin test by the Goodman method, it was found that the proportion of children who had taken three or more doses of BCG by oral administration and showed strong reaction to the tuberculin test is significantly greater than that observed for the non-reactors, a fact which does not hold true for the one to four age group. For the children who had taken one or two doses there was no significant statistical difference.

UNITERMS: Tuberculosis in childhood. Tuberculin test. BCG vaccination, oral.

\section{REFERENCIAS BIBLIOGRAFICAS}

1. ALMEIDA, E. S. Infeç̧ăo tuberculosa natural e o uso do BCG oral e intradérmico em escolares de Laranjal Paulista, SP, Brasil. Rev. Saude públ., S. Paulo, 7:189-97, 1973.

2. ALMEIDA, E. S. et al. Nível de infecção tuberculosa e viragem tuberculínica pelo BCG intradérmico em escolares de Botucatu, São Paulo, em 1969. Rev. Saúde públ., S. Paulo, 8:31-41, 1974.

3. AMARAL, J. P. \& SOERENSEN, B. BCG por via oral em cobaios. Hospital, Rio de Janeiro, 58:1053-7, 1960 .

4. ASSIS, A. Alergia tuberculínica depois da vacinação oral em recém-nascidos com dose única $(100 \mathrm{mg})$ de BCG. Hospital, Rio de Janeiro, 33:169-77, 1948.

5. ASSIS, A. \& CARVALHO, A. Vacinação BCG por via bucal e alergia tuberculínica. Hospital, Rio de Janeiiro, $18: 543-53,1940$.

6. BROLIO, R. Controle de duas epidemias de tuberculose em oriancas merores de
5 anos de idade vacinadas com $B C G$ oral, ocorridas nos anos de 1967 e 1969, numa creche no Municipio de são Paulo. Săo Paulo, 1972. [Tese de Doutoramento - Faculdade de Saúde Pública da USP].

7. BRólIO, $R$. et al. Hipersensibilidade tuberculínica em crianças menores de um ano de idade vacinadas com BCG oral. Rev. Saúde públ., S. Paulo, 11: 111-7, 1977.

8. CAMPANHA NACIONAL CONTRA A TUBERCULOSE. Comissão Técnica. Prova tuberculínica em Saúde Pública (2a. recomendação). Rev. Serv. nac. Tuberc., $12: 219-30,1968$.

9. CERTAin, D. A. et al. Análise dos resultados da pesquisa da infecção tuberculosa e do primeiro programa de vacinação pelo $B C G$ intradérmico em escolares de São Paulo, Brasil, 19711974. Rev. Saude públ., S. Paulo, 9: 125-36, 1975 . 
DIAS, M. H. de P. \& HAYASHI, A. Prova tuberculínica, BCG oral e infecção tuberculosa em crianças menores de 5 anos. Rev. Saúde públ., S. Paulo, 12:443-54, 1978.

10. CERTAIN, D. A. et al. Impregnação tuberculosa em menores de 14 anos detectada pela prova tuberculinica em 2 áreas do Município de São Paulo. Rev. Saúde públ., S. Paulo, 5:51-8, 1971.

11. COMISSÃO de BCG: vacinação BCG no Brasil (Politica dia Divisão Nacional de Tuberculose), Rev. Div. nac. Tuberc, 17:255-62, 1973.

12. GOODMAN, L. A. Simultaneous confidence intervals for contrasts among multinomial populations. Ann. Math. Statist., $35: 716-25,1964$.

13. HORWITZ, O. \& BUNCH-CHRISTENSEN, $\mathrm{K}$. Correlation between tuberculin sensitivity after 2 months and 5 years among BCG vaccinated subjects. Bull. Wld Hith Org., 47:49-58, 1972.

14. KAHN, H. et al, BCG vaccination: a comparison of post-vaccination tuberculin sensitivity after oral and intradermal vaccination of newborn infants. Tubercle, 51:423-9, 1970.

15. NUNES, R. L. Prevalência de tuberculose: infecção e doença numa àrea do $\mathrm{Mu-}$ nicipio de Osasco, SP. São Paulo. 1970. [Dissertação de Mestrado - Fa. culdade de Saúde Pública da USP]

16. IIMA. I. L. et al. Ação programada de vacinação BCG intradérmico. Rev. Dit. nac. Tuberc., 16:12-64, 1972.

17. LOUREIRO, $\mathbf{S}$, et al. Teste tuberculínico em área urbana. Rev. Div. nac. Tuberc., 17:79-84, 1978.

18. PASSOS FILHO, M. C. R. et al. Vacinação BCG oral em escolares de São Bernardo do Campo: levantamento prévio dos reatores e não reatores aos testes tuberculínicos. Hospital, Rio de Janeiro, 79:243-57, 1971.

19. PASSOS FILHO, M. C. R. et al. BCG pelo método oral (Alergia pós-vacinal em recém-nascidos e em escolares). Indice de infeçãa tuberculosa em escolares. Rev, Div. nac, Tuberc., 15:271-80, 1971

20. PAZ DE ALMEIDA, A. Prevalência da infecção tuberculosa em escolares das capitais brasileiras. Rev. Div. nac. Tuberc, 18:413-44, 1974.

21. PUSCH, C. M. et al. Cadastramento tuberculínico de escolares em Diadema. Rev. Div. nac. Tuberc., 19:128-35. 1975.

22. RUFFino NetTo, A. et al. Alergia tuberculínica pós-vacinação com BCG intradérmico e pós-infecção natural. Rev Div. nac. Tuberc., 20:18-27, 1976.

23. SÉNÉCAL, M. J. Sur la raccination BCG par voie buccale. IV. Resultats. A Allergie vaccinale. Rev. Tuberc., Paris, $25: 512-3,1961$.

24. SOERENSEN, B, et al, Conversão tuberculínica da vacina BCG administrada pelas vias intradérmicas, oral e multipuntura, em cobaios. [Trabalho apresentado ao $7 \%$ Congresso de Microbiologia, Porto Alegre, 1976]

25. TOSSIN, L, N. Teste tuberculínico e prevalência de infecção tuberculosa em escolares de Porto Alegre. Rev. Div. nac. Tuberc., $16: 112-22,1972$.

Recebido para publicacao em 07/04/1978. Aprovado para publicacto em 18/04/1978. 